\title{
An evaluation of the 2016 influenza vaccination in pregnancy campaign in NSW, Australia
}

\author{
Samantha Carlsone, ${ }^{a, b, d}$, Aditi Dey ${ }^{a, c}$ and Frank Beard ${ }^{a, b}$ \\ a National Centre for Immunisation Research and Surveillance, Sydney, NSW, Australia \\ b School of Public Health, Faculty of Medicine and Health, University of Sydney, NSW, Australia \\ c Discipline of Child and Adolescent Health, Faculty of Medicine and Health, University of Sydney, NSW, Australia \\ d Corresponding author: samantha.carlson@health.nsw.gov.au
}

\section{Article history}

Publication date: March 2020

Citation: Carlson S, Dey A, Beard F.

An evaluation of the 2016 influenza vaccination in pregnancy campaign in NSW, Australia. Public Health Res Pract. 2020;30(1):e29121908. First published 2 May 2019. https://doi.org/10.17061/ phrp29121908

\section{Key points}

- An evaluation of the 2016 New South Wales (NSW) Health influenza vaccination in pregnancy public information campaign shows that engagement with campaign messages on social media was low, and traditional media activity was highest during the peak influenza season

- Estimated uptake of influenza vaccination by pregnant women in NSW was $54 \%$ in 2016. Compared to an uptake of $17 \%$ in a similar population in 2011 , this is an increase but still suboptimal

- Pregnant women turn to multiple sources for information about influenza vaccination in pregnancy, but a strong recommendation from a healthcare provider is an important determinant of vaccine uptake

- Many pregnant women have unresolved concerns about influenza vaccination, and more detailed information in campaign materials about vaccination effectiveness and safety for mother and baby could be beneficial

\section{Abstract}

Objectives and importance: Influenza vaccination in pregnancy has been funded under the Australian National Immunisation Program since 2010, yet uptake is suboptimal. We evaluated the 2016 New South Wales (NSW) Health influenza vaccination in pregnancy public information campaign.

Study type: Mixed methods.

Methods: We searched Factiva, ProQuest Global Newsstream and Google News for news articles relevant to the 2016 campaign. We reviewed information provided by NSW Health about NSW Health-initiated social media activity relevant to the campaign, and also assessed engagement with campaign messages using publicly available information. Between September and October 2016, we surveyed pregnant women at the antenatal clinic of a large tertiary hospital in Sydney to estimate influenza vaccine uptake during pregnancy, identify key information sources, and understand knowledge and attitudes regarding influenza vaccination.

Results: Thirty-one news articles were published in traditional media between April and August 2016. A single campaign advertisement on the NSW Health Facebook page had 449609 impressions, with a click-through rate to the NSW Health website of $0.4 \%$. Sixteen sponsored articles were posted on Facebook and six sponsored images were posted on Instagram, with relatively limited engagement.

A total of 71 pregnant women participated in the survey. Of these, 90\% were aware that pregnant women can receive an influenza vaccine during pregnancy, with 54\% reporting that they had been vaccinated in their current pregnancy. Fifty-five per cent had read a brochure about influenza vaccination in pregnancy. Many women, particularly those who were unvaccinated, were uncertain about the effectiveness and safety of influenza vaccination in pregnancy.

Conclusion: Both traditional and social media platforms can be used to promote influenza vaccination for pregnant women, although there are challenges to achieving high reach of messages via both methods. Selfreported vaccination coverage in our study was higher than previous NSW estimates, but still suboptimal. Further efforts are required to understand and address provider and patient attitudes and concerns about influenza 
vaccination in pregnancy, especially regarding effectiveness and safety. Our findings and recommendations should be of interest to healthcare providers and public health agencies Australia-wide, to inform campaigns and programs to improve uptake of antenatal influenza vaccination.

\section{Introduction}

Pregnant women are at increased risk of suffering serious complications from influenza. ${ }^{1}$ Influenza can also have adverse effects on the fetus, and increases the risk of premature birth, stillbirth and pregnancy loss. ${ }^{1,2} \mathrm{~A}$ variety of overseas ${ }^{3,4}$ and Australian studies ${ }^{5-8}$ have shown that vaccinating pregnant women is safe and effective in reducing these complications.

Pregnant women have been classified as the group of highest priority for seasonal influenza vaccination programs by the World Health Organization since 2012. ${ }^{9}$ Influenza vaccination in pregnancy has been recommended by the Australian Technical Advisory Group on Immunisation since March 2000, and has been funded under the National Immunisation Program since January 2010. ${ }^{10}$ Vaccination is available through general practitioners (GPs) and some antenatal clinics.

Despite being strongly recommended, vaccine uptake is suboptimal. Factors identified as contributing to poor uptake include lack of awareness by pregnant women, lack of provider recommendation, and maternal concerns

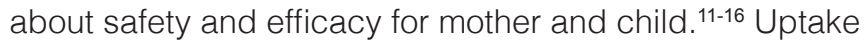
has been particularly poor in New South Wales (NSW), with studies estimating that $25-27 \%$ of pregnant women were vaccinated against influenza during their pregnancy in 2011-2012. ${ }^{13,17}$ This was less than the estimated national coverage of $34 \%$ in $2012-2014 .^{8}$

To improve uptake, the NSW Department of Health (NSW Health) first implemented a public information campaign in 2014. This ongoing and annual campaign delivers information about the benefits, safety and effectiveness of influenza vaccination in pregnancy through three different brochures, traditional media (television and newspapers) and social media (Facebook and Instagram).

We aimed to evaluate the 2016 public information campaign to assess its reach and to identify issues that could be addressed in future campaigns to increase vaccination uptake. As part of this evaluation, we aimed to estimate influenza vaccination coverage in pregnant women and to determine their information sources, knowledge and attitudes about influenza vaccination and disease, to inform future campaigns.

\section{Methods}

Our evaluation methodology had two main components: assessing the promotion of the campaign through traditional and social media, and a questionnaire administered to a cohort of pregnant women.

\section{Media search strategy}

\section{Online databases}

Key articles that disseminated information about the campaign were identified through searches on Factiva, ProQuest Global Newsstream and Google News. On 1 September 2016, articles published between 1 January and 31 August 2016 were searched by keywords to identify relevant articles that included variations of flu, vaccin*, pregnan*, women and NSW. Articles were included for analysis if the primary message of the article was a recommendation from NSW Health for pregnant women to be vaccinated against influenza.

\section{Social media}

Staff from the Strategic Relations and Communications Unit at NSW Health provided information about NSW Health-initiated social media activity relevant to the 2016 campaign: an advertisement on Facebook, and sponsored Facebook articles and Instagram posts. This included the impressions and reach available for the Facebook articles as of 14 September 2016, and impressions, clicks on the advertisement and clicks through to the website from the Facebook advertisement as of 22 September 2016. Publicly available information about engagements with Facebook articles (likes and shares) and Instagram posts (likes) was accessed on 14 September 2016.

The reach of and engagement with the campaign advertisement, Facebook articles and Instagram posts was assessed. Facebook articles and Instagram posts were categorised by whether they were solely about the health of the mother, the baby, or both the mother and the baby. Comments made in response to advertisements, articles or images were not assessed because these were curated by the administrators of the social media pages.

\section{Questionnaire methodology}

\section{Study design}

A self-administered questionnaire distributed to pregnant women.

\section{Questionnaire development and content}

The questionnaire was developed using modified questions from a questionnaire used in a similar population in $2011^{18}$, and the information from NSW Health brochures about influenza vaccination in pregnancy. Construct validity was tested with seven mothers, and the survey was modified where required. 
The key domains of the questionnaire included questions about demographics, information sources, feedback on the brochures developed by NSW Health, and participants' past or intended influenza vaccination uptake. Images of the different brochure covers were used adjacent to relevant questions to assist in memory recall. Four versions of the final questionnaire with differing question order were randomly distributed to participants to minimise social desirability bias. Selfreported vaccination status was also collected and validated (when possible) by assessing the participant's handheld antenatal card to confirm receipt of vaccine and date of receipt.

\section{Study population and participant recruitment}

A convenience sample of English-speaking pregnant women aged over 18 years and attending the antenatal clinic of a large (5500 births/year) tertiary hospital in western Sydney, NSW, were approached during September and October 2016.

\section{Data analysis}

Descriptive statistics were calculated on unweighted data using Microsoft Excel.

\section{Ethics approval}

Ethics approval for this mixed-methods evaluation was gained from the Sydney Children's Hospitals Network Human Research Ethics Committee (LNR/16/SCHN/275).

\section{Results}

\section{Media promotion}

\section{News articles}

Combined database searches yielded 212 articles, of which 31 (15\%) were news articles relevant to the NSW Health campaign. Promotion of the public health message began in April 2016, with a total of six relevant news articles published in April (Figure 1), two of which immediately followed a press release issued by NSW Health on 26 April 2016. ${ }^{19}$ Eight articles were published between May and July, during which the campaign focused on promoting the message on social media. Following another press release issued by NSW Health on 16 August 2016²0 17 news articles were published.

\section{Facebook advertisement}

On 17 May 2016, a campaign advertisement targeting pregnant women in NSW was placed on the NSW Health Facebook page. It was an active advertisement on the page until 31 July. After this, it did not actively target an audience, but remained viewable. The key message was: "The risk of premature labour and delivery is increased in pregnant women with the flu. Don't risk it. Speak to your GP about a free flu shot." The advertisement contained a link that led to a NSW Health website with more detailed information.

Over 128 days, including 75 days of active targeting, the advertisement had 449609 impressions and the link to the NSW Health website was clicked on 1875 times, giving a click-through rate of $0.4 \%$.

Figure 1. Timeline of media activity from April to August 2016 related to the 2016 NSW Health influenza vaccination in pregnancy campaign

\begin{tabular}{|c|c|c|c|c|c|c|}
\hline April & $\begin{array}{l}\text { News article } \\
\times 4\end{array}$ & $\begin{array}{l}\text { NSW Health } \\
\text { media } \\
\text { release }\end{array}$ & $\begin{array}{l}\text { News article } \\
\times 2\end{array}$ & & & \\
\hline May & $\begin{array}{l}\text { News article } \\
\times 1\end{array}$ & $\begin{array}{l}\text { Facebook } \\
\text { article } \times 1\end{array}$ & $\begin{array}{l}\text { News article } \\
\times 2\end{array}$ & $\begin{array}{l}\text { Instagram } \\
\text { post x } 1\end{array}$ & & \multirow{3}{*}{$\begin{array}{l}\text { Facebook } \\
\text { advertisement }\end{array}$} \\
\hline June & $\begin{array}{l}\text { News article } \\
\times 2\end{array}$ & $\begin{array}{l}\text { Facebook } \\
\text { article } \times 7\end{array}$ & $\begin{array}{l}\text { Instagram } \\
\text { post } \times 2\end{array}$ & $\begin{array}{l}\text { Facebook } \\
\text { article } \times 4\end{array}$ & $\begin{array}{l}\text { Instagram } \\
\text { post x } 1\end{array}$ & \\
\hline July & $\begin{array}{l}\text { Facebook } \\
\text { article } \times 1\end{array}$ & $\begin{array}{l}\text { Instagram } \\
\text { post } \times 2\end{array}$ & $\begin{array}{l}\text { Facebook } \\
\text { article } \times 3\end{array}$ & $\begin{array}{l}\text { News article } \\
\times 3\end{array}$ & & \\
\hline
\end{tabular}

\begin{tabular}{|l|l|l|}
\hline August & $\begin{array}{l}\text { NSW Health } \\
\text { media } \\
\text { release }\end{array}$ & $\begin{array}{l}\text { News article } \\
\times 17\end{array}$ \\
\hline
\end{tabular}




\section{Facebook articles}

NSW Health paid for six individual articles that promoted influenza vaccination in pregnancy to be posted 2-3 times each between May and July 2016 across two Facebook pages belonging to a mainstream media outlet targeting women (Figure 1). The combined number of followers of these pages on the final day of data collection (14 September 2016) was 2066 933. Five articles were categorised as providing information about the health of the mother and the baby, and one provided information solely about the baby. On average, each article about the health of both the mother and the baby was liked 31 times and reached 59598 Facebook users; the article about the health of the baby was liked 12 times and reached 30295 people.

\section{Instagram posts}

Six images relating to influenza vaccination in pregnancy were posted on an Instagram page of the mainstream media outlet targeting women between May and July 2016 (Figure 1). The number of followers of the Instagram page on the final date of data collection (14 September 2016) was 76400 . Two posts were about the health of the mother and the baby, three were about the mother, and one was about the baby. On average, each post about the health of the mother and baby had 193 likes, and each post about the health of the mother had 170 likes. The single post about the health of the baby had 151 likes.

\section{Questionnaire}

A single researcher (SC) approached 122 women in the antenatal clinic waiting room, of whom 103 (84\%) were eligible to participate in the survey. Of the 19 women who were ineligible, 14 (74\%) did not speak English and $5(26 \%)$ were not pregnant. Of eligible women, $69 \%$ (71/103) participated. Reasons for nonparticipation in the eligible group were: being called into their appointment before beginning the survey $(9,28 \%)$; being cautious about time (1, $3 \%)$; or no reason given $(22,69 \%)$.

The majority of participants were aged between 25 and 34 years (68\%) and in their third trimester of pregnancy (62\%) (Table 1). Fifty-nine per cent of women had been pregnant before. Nearly all participants (99\%) lived in NSW, with $97 \%$ of these women living in the western suburbs of Sydney. Two respondents identified as being of Aboriginal or Torres Strait Islander origin. Nearly one-quarter $(24 \%)$ had completed a postgraduate degree. Just over one-third (38\%) spoke only English at home (Table 1).

The self-reported uptake of influenza vaccine was $54 \%(38 / 71)$. Just under half $(35 / 71,49 \%)$ of the participants had their antenatal card available for review. Self-reported vaccination status matched the documentation on the antenatal card in approximately two-thirds $(22 / 35,63 \%)$ of these women; one reported not being vaccinated when their card said they had been, and $12(34 \%)$ reported receiving an influenza vaccine during the pregnancy but this was not documented on their antenatal card. Some of these women provided reasons for the lack of documentation, including that their healthcare provider did not record it or they were vaccinated at work but did not have their card with them at the time. The median gestational age of vaccine receipt for women with antenatal cards available was 28 weeks (range 16-31 weeks). Of the women who reported being vaccinated, 39\% (15/38) had never received an influenza vaccine before being pregnant. Seventeen per cent of participants (12/71) reported they still planned to be vaccinated during their pregnancy; most of these women $(10 / 12,83 \%)$ were in their first or second trimester.

Most participants $(90 \%, 64 / 71)$ were aware of the recommendation that pregnant women receive an influenza vaccination in pregnancy; 55\% (35/64) of these women had already been vaccinated. Midwives were the most common source of information (47/64); however, only $51 \%$ (24/47) of women who had discussed influenza vaccination with a midwife had been vaccinated (Figure 2). GPs were also a major source of information, with 43/64 women discussing influenza vaccination with a GP; almost two-thirds of these women reported being vaccinated $(27 / 43,63 \%)$. Another major source of information was the internet. One in two women (32/64) who were aware of the recommendation to have an influenza vaccination reported that they had seen the advice on the internet; however, more than half (18/32, $56 \%$ ) of these women remained unvaccinated.

A brochure was a source of information for just over half $(35 / 64,55 \%)$ of participants who were aware of influenza vaccination in pregnancy, and 18 of these women (51\%) had been vaccinated (Figure 2). Just over three-quarters $(27 / 35,77 \%)$ of the women who had read about influenza vaccination in a brochure had read at least one of the three brochures developed by NSW Health, and $63 \%(17 / 27)$ of these women reported that the information in the brochures helped them to make a decision about

Figure 2. Pregnant women's sources of information about influenza vaccination in pregnancy $(n=64)$

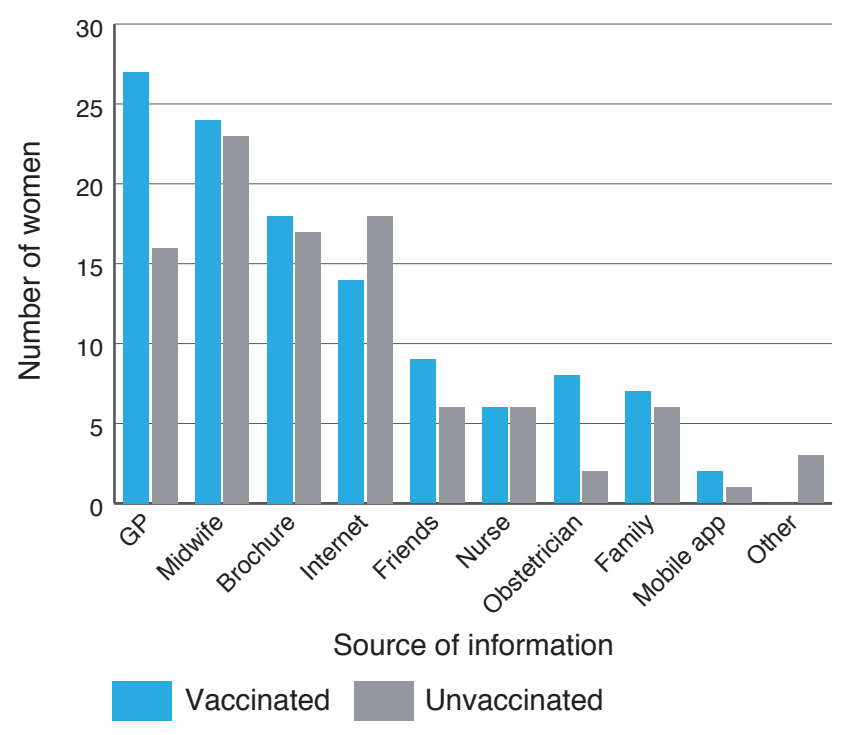


Table 1 Characteristics of participants by influenza vaccination status

\begin{tabular}{|c|c|c|}
\hline Characteristic & $\begin{array}{c}\text { Total number } \\
n(\%)\end{array}$ & $\begin{array}{c}\text { Number vaccinated } \\
n(\%)\end{array}$ \\
\hline Number surveyed & $71(100)$ & $38(54)$ \\
\hline
\end{tabular}

\section{Age (years)}

$\begin{array}{rrr}18-24 & 11(15) & 8(73) \\ 25-34 & 48(68) & 26(54) \\ 35-44 & 12(17) & 4(33)\end{array}$

\section{Trimester}

$\begin{array}{lcr}\text { First (0-12 weeks) } & 3(4) & 0(0) \\ \text { Second (13-27 weeks) } & 24(34) & 7(29) \\ \text { Third (28-40 weeks) } & 44(62) & 31(70)\end{array}$

\section{First pregnancy}

$\begin{array}{lll}\text { Yes } & 29(41) & 20(69)\end{array}$

No

$42(59)$

$18(43)$

\section{Aboriginal status}

Neither Aboriginal nor Torres Strait Islander

$69(97)$

Aboriginal

$1(1)$

Torres Strait Islander

$0(0)$

Aboriginal and Torres Strait Islander

$1(1)$

NA

1 (100)

\section{Highest level of education}

High school

Certificate or diploma

Undergraduate degree

Postgraduate degree

\section{Language(s) spoken at home}

English

English and another language

$17(24)$

$9(53)$ vaccination. These women were either vaccinated (15/17, $88 \%)$ or planning to be vaccinated $(2 / 17,12 \%)$.

Of those who were vaccinated, 95\% (36/38) agreed or strongly agreed that influenza vaccination in pregnancy is safe for the mother (Figure 3a), and 79\% (30/38) agreed that it is protective for the mother. Seventyone per cent (27/38) agreed or strongly agreed that influenza vaccination in pregnancy will protect the fetus. Forty-seven per cent (18/38) disagreed or strongly disagreed with the statement that influenza vaccination will not protect the newborn. Seventy-nine per cent (30/38) disagreed or strongly disagreed that influenza vaccination in pregnancy is dangerous for the baby.

Of those who were not vaccinated, 48\% (16/33) agreed or strongly agreed with the statement that influenza vaccination in pregnancy is safe for the mother (Figure 3b), and 39\% (13/33) agreed that it is protective for the mother. Fifty-two per cent (17/33) agreed or strongly agreed that influenza vaccination in pregnancy will protect the fetus. Thirty-three per cent (11/33) disagreed or strongly disagreed with the statement that influenza vaccination will not protect the newborn. Fortyfive per cent (15/33) disagreed or strongly disagreed that influenza vaccination in pregnancy is dangerous for the baby. A greater proportion of participants who were not vaccinated did not answer the questions assessing knowledge and attitudes, compared to those who were vaccinated, which may reflect greater levels of uncertainty among this group. 
Figure 3. Pregnant women's knowledge and perceptions of influenza disease and influenza vaccination in pregnancy, by influenza vaccination status

(a) Vaccinated women $(n=38)$

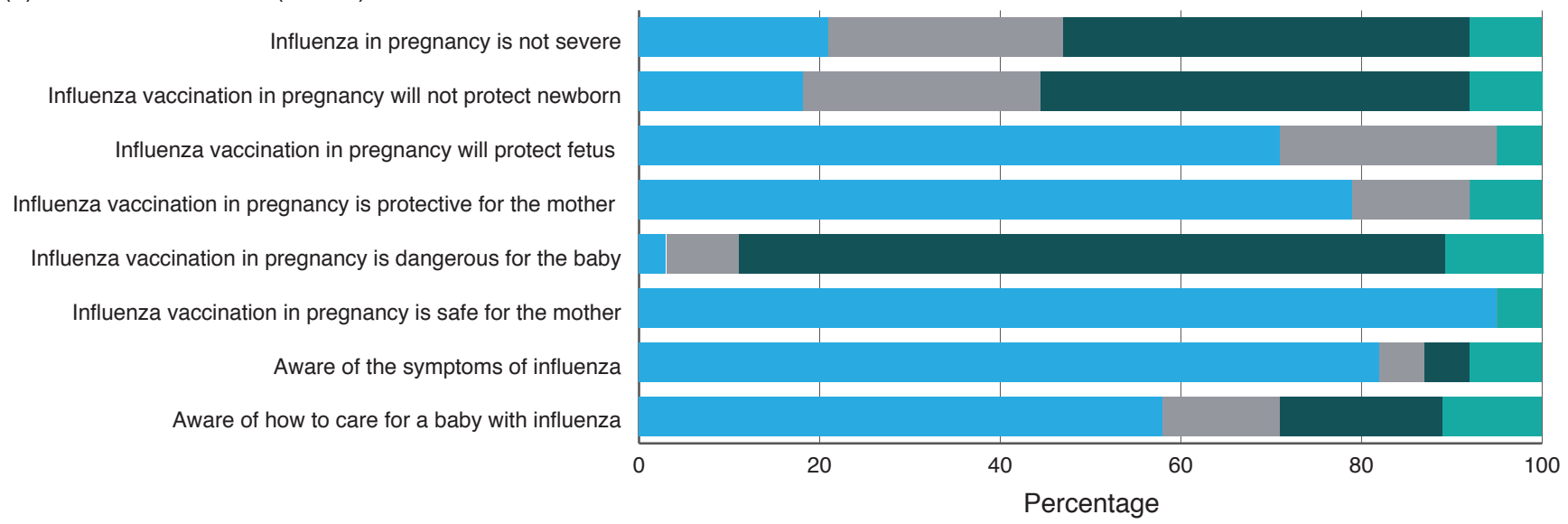

(b) Unvaccinated women $(n=33)$

Influenza in pregnancy is not severe

Influenza vaccination in pregnancy will not protect newborn

Influenza vaccination in pregnancy will protect fetus

Influenza vaccination in pregnancy is protective for the mother

Influenza vaccination in pregnancy is dangerous for the baby

Influenza vaccination in pregnancy is safe for the mother

Aware of the symptoms of influenza

Aware of how to care for a baby with influenza

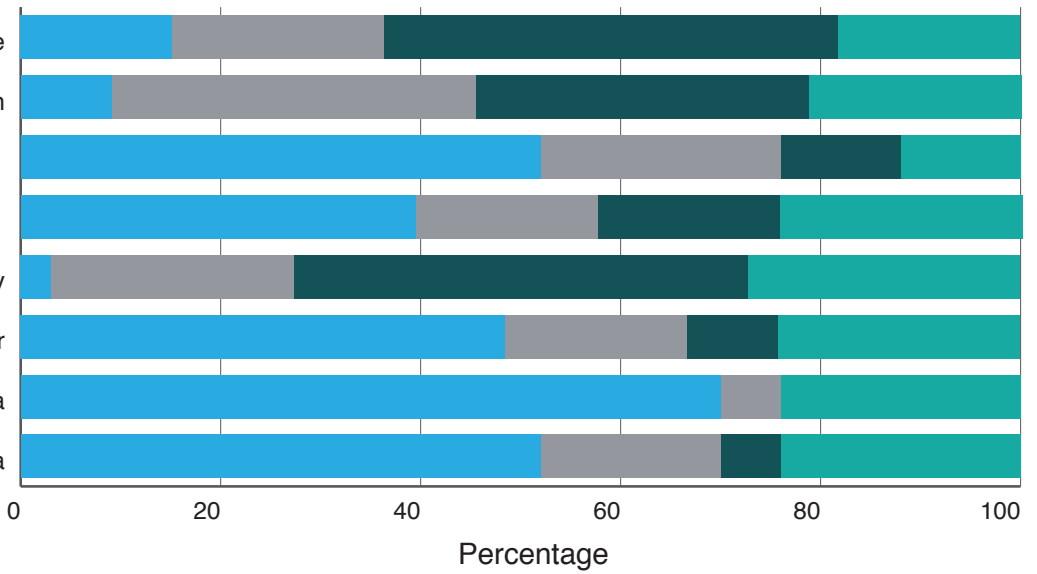

Agree-Strongly agree Neither agree nor disagree

Disagree-Strongly disagree No answer

\section{Discussion}

We found considerably more traditional media coverage of the 2016 NSW influenza vaccination in pregnancy campaign after the second NSW Health press release in August 2016, which described a 'spike' in influenza cases and outlined what was happening in vulnerable populations due to influenza ${ }^{20}$, compared with the first press release in April 2016, which focused on what can happen to pregnant women if they get influenza while pregnant. ${ }^{19}$ Traditional media is a key source of information about influenza vaccination in pregnancy for Australian women. ${ }^{12,13,21,22}$ Press releases issued before the peak influenza season are warranted to raise awareness and encourage vaccine uptake. However, they may struggle to achieve high media uptake and so should be followed with later media releases that highlight increased influenza activity; later releases are more readily personalised and likely to achieve greater media uptake.
Because it is difficult to achieve traditional media coverage of immunisation messages before the start of the influenza season, social media may be an appealing alternative communication channel. Although it is difficult to determine the impact of a social media campaign on the health behaviour of social media users, particularly when only assessing interactions with content such as 'likes' and 'shares'23,24, our results show that engagement with the social media content for this campaign was poor. However, social media content that related to the health of both mother and baby was more frequently engaged with than content related solely to the health of the baby. Given that pregnant women have been shown to perceive influenza as more of a risk to themselves than their baby $^{22}$, campaigns should include information about the risks to both mother and baby in a single resource.

We suggest that public health campaigns that aim to use social media could attract social media users to their content by having engaging and clear information that 
leads users to click on the content for more information. Social media campaigns should also be interactive, by inviting viewers to like, comment on or share the content, because this may generate a larger reach and potentially higher click-through rate. ${ }^{23}$ Multipronged social media strategies using several tools as complementary approaches can reinforce and increase the impact of social media campaigns on behaviour change. ${ }^{23,24}$

As done in this study, evaluations of public health campaigns with a social media component should survey the intended audience of the campaign. However, such surveys should include questions to specifically determine whether those who seek information online actually encountered the social media campaign, and, if so, whether it influenced the desired behavioural outcome.

Our survey of pregnant women found self-reported influenza vaccination uptake to be $54 \%$. Although still suboptimal, influenza vaccine uptake among pregnant women is increasing. The rate in this study was more than three times the rate (17\%) seen in a study from the same hospital in $2011^{17}$, and two-thirds higher than the rate $(34 \%)$ observed in a large national cohort study of pregnant women between 2012 and 2014. ${ }^{8}$ This increase is also confirmed by another large national study, which estimated influenza vaccine uptake to be $46 \%$ in 2015-2016. ${ }^{15}$

Only half of the women in our study who had discussed influenza vaccination with a midwife were vaccinated, compared to almost two-thirds of those who had discussed it with a GP. This could reflect lower levels of recommendations by midwives and/ or logistical issues (women in our study could not be vaccinated directly by their midwife and needed to make a separate appointment with an authorised nurse immuniser at the associated immunisation clinic). A strong recommendation from a healthcare provider is the most important determinant of influenza vaccine uptake by pregnant Australian women. ${ }^{11-14,16}$ A 2012 study assessing the knowledge, attitudes, beliefs and practices of GPs in Sydney found that one-third did not consider influenza during pregnancy to be a serious risk for the mother or the baby, and more than half had significant concerns about the safety of influenza vaccination in pregnancy. ${ }^{25}$ Further studies to assess whether midwives also hold these concerns are warranted, to inform tailored programs to improve midwives' knowledge of, confidence in and advocacy for vaccination during pregnancy.

A substantial proportion of unvaccinated women indicated concern or uncertainty about the safety and effectiveness of influenza vaccination in pregnancy. Additionally, some vaccinated participants expressed uncertainty over the effectiveness of influenza vaccination in protecting the fetus and newborn baby. More detailed information in campaign materials about effectiveness and safety could be beneficial.
Our pilot survey had a small sample size, was limited to English-speaking participants from one hospital and was conducted relatively late in the influenza season. Future surveys should start recruitment earlier and across multiple sites. Consideration should also be given to inclusion of non-English speaking participants, as knowledge, attitudes, behaviours and concerns about immunisation may differ in this group.

\section{Conclusion}

Both traditional and social media platforms can be used to promote influenza vaccination of pregnant women, although there are challenges to achieving high reach of messages via both methods. Self-reported vaccination coverage in our study was higher than previous NSW estimates, but still less than optimal. Further efforts are required to understand and address provider and patient attitudes and concerns about influenza vaccination in pregnancy, especially regarding effectiveness and safety. Our findings and recommendations should be of interest to healthcare providers and public health agencies Australia-wide, to inform campaigns and programs that seek to improve the uptake of antenatal influenza vaccination

\section{Acknowledgements}

This study was conducted under the National Centre for Immunisation Research and Surveillance's ongoing funding agreement with NSW Health to undertake evaluations of immunisation programs.

We acknowledge Kerrie Wiley of the University of Sydney and Robin Gilmour of NSW Health for their assistance in the survey development. We also thank the staff of the Strategic Relations and Communications Unit of NSW Health, and the staff of the Westmead Hospital Antenatal Clinic.

\section{Peer review and provenance}

Externally peer reviewed, not commissioned.

\section{Competing interests}

None declared.

\section{Author contributions}

All authors contributed to the study design. SC was responsible for data collection and management. SC and $A D$ analysed the data. SC prepared the manuscript and $A D$ and $F B$ provided feedback. 


\section{References}

1. Tamma PD, Steinhoff MC, Omer SB. Influenza infection and vaccination in pregnant women. Expert Rev Respir Med. 2010;4(3):321-8.

2. Rasmussen SA, Jamieson DJ, Uyeki TM. Effects of influenza on pregnant women and infants. Am J Obstet Gynecol. 2012;207(3):S3-8.

3. Zaman K, Roy E, Arifeen SE, Rahman M, Raqib R, Wilson $\mathrm{E}$, et al. Effectiveness of maternal influenza immunization in mothers and infants. N Engl J Med. 2008;359(15):1555-64.

4. Shakib JH, Korgenski K, Presson AP, Sheng X, Varner MW, Pavia AT, et al. Influenza in infants born to women vaccinated during pregnancy. Pediatrics. 2016:e20152360.

5. Regan AK, Tracey LE, Blyth CC, Richmond PC, Effler PV A prospective cohort study assessing the reactogenicity of pertussis and influenza vaccines administered during pregnancy. Vaccine. 2016;34(20):2299-304.

6. Regan AK, Moore HC, de Klerk N, Omer SB, Shellam G, Mak DB, et al. Seasonal trivalent influenza vaccination during pregnancy and the incidence of stillbirth: population-based retrospective cohort study. Clin Infect Dis. 2016;62(10):1221-7.

7. Regan AK, de Klerk N, Moore HC, Omer SB, Shellam G, Effler PV. Effectiveness of seasonal trivalent influenza vaccination against hospital-attended acute respiratory infections in pregnant women: a retrospective cohort study. Vaccine. 2016;34(32):3649-56.

8. McHugh L, Andrews RM, Lambert SB, Viney KA, Wood N, Perrett KP, et al. Birth outcomes for Australian motherinfant pairs who received an influenza vaccine during pregnancy, 2012-2014: the FluMum study. Vaccine. 2017;35(10):1403-9.

9. World Health Organization. WHO recommends seasonal influenza vaccination to pregnant women as the highest priority; 2012 Nov 23 [cited 2018 Jan 3]. Available from: www.who.int/immunization/newsroom/newsstory_ seasonal_influenza_vaccination_pregnancy/en/

10. National Centre for Immunisation Research and Surveillance. Significant events in influenza vaccination practice in Australia. Sydney: NCIRS; 2018 [cited 2019 Apr 8]. Available from: www.ncirs.org.au/sites/default/ files/2018-11/Immunisation-policy-and-practice-AustraliaJuly-2018.pdf

11. McCarthy EA, Pollock WE, Tapper L, Sommerville M, McDonald S. Increasing uptake of influenza vaccine by pregnant women post $\mathrm{H} 1 \mathrm{~N} 1$ pandemic: a longitudinal study in Melbourne, Australia, 2010 to 2014. BMC Pregnancy Childbirth. 2015;15(1):53.
12. Mak DB, Regan AK, Joyce S, Gibbs R, Effler PV. Antenatal care provider's advice is the key determinant of influenza vaccination uptake in pregnant women. Aust N Z J Obstet Gynaecol. 2015;55(2):131-7.

13. Maher L, Hope K, Torvaldsen S, Lawrence G, Dawson A, Wiley $\mathrm{K}$, et al. Influenza vaccination during pregnancy: coverage rates and influencing factors in two urban districts in Sydney. Vaccine. 2013;31(47):5557-64.

14. Taksdal SE, Mak DB, Joyce S, Tomlin S, Carcione D, Armstrong PK, et al. Predictors of uptake of influenza vaccination: a survey of pregnant women in Western Australia. Aust Fam Physician. 2013;42(8):582-6.

15. Danchin M, Costa-Pinto J, Attwell K, Willaby H, Wiley K, Hoq $\mathrm{M}$, et al. Vaccine decision-making begins in pregnancy: correlation between vaccine concerns, intentions and maternal vaccination with subsequent childhood vaccine uptake. Vaccine. 2018;36(44):6473-9.

16. Regan AK, Mak DB, Hauck YL, Gibbs R, Tracey L, Effler PV. Trends in seasonal influenza vaccine uptake during pregnancy in Western Australia: implications for midwives. Women Birth. 2016;29(5):423-9.

17. Wiley KE, Massey PD, Cooper SC, Wood NJ, Ho J, Quinn HE, et al. Uptake of influenza vaccine by pregnant women: a cross-sectional survey. Med J Aust. 2013;198(7):373-5.

18. Wiley K, Massey P, Cooper S, Wood N, Quinn H, Leask J. Pregnant women's intention to take up a post-partum pertussis vaccine, and their willingness to take up the vaccine while pregnant: a cross sectional survey. Vaccine. 2013;31(37):3972-8.

19. NSW Government. NSW Health urges pregnant women to have free flu vaccination early. Sydney: NSW Health; 26 April 2016 [cited 2018 Jan 3]. Available from: www.health. nsw.gov.au/news/Pages/20160426_00.aspx

20. NSW Government. Protect vulnerable people as flu cases rise. Sydney: NSW Health; 16 Aug 2016 [cited 2018 Jan 3]. Available from: www.health.nsw.gov.au/news/ Pages/20160816_00.aspx

21. McCarthy EA, Pollock WE, Nolan T, Hay S, McDonald S. Improving influenza vaccination coverage in pregnancy in Melbourne 2010-2011. Aust N Z J Obstet Gynaecol. 2012;52(4):334-41.

22. Wiley KE, Cooper SC, Wood N, Leask J. Understanding pregnant women's attitudes and behavior toward influenza and pertussis vaccination. Qual Health Res. 2015;25(3):360-70.

23. Freeman B, Potente S, Rock V, Mclver J. Social media campaigns that make a difference: what can public health learn from the corporate sector and other social change marketers. Public Health Res Pract. 2015;25(2):e2521517. 
24. Korda H, Itani Z. Harnessing social media for health promotion and behavior change. Health Promot Pract. 2013;14(1):15-23.
25. Maher L, Dawson A, Wiley K, Hope K, Torvaldsen S, Lawrence $G$, et al. Influenza vaccination during pregnancy: a qualitative study of the knowledge, attitudes, beliefs, and practices of general practitioners in Central and South-Western Sydney. BMC Fam Pract. 2014;15(1): 102 .

\section{Copyright: (c) (i) (2)}

(C) 2019 Carlson et al. This article is licensed under the Creative Commons Attribution-NonCommercial-ShareAlike 4.0 International Licence, which allows others to redistribute, adapt and share this work non-commercially provided they attribute the work and any adapted version of it is distributed under the same Creative Commons licence terms. See: www.creativecommons.org/licenses/by-nc-sa/4.0/ 